\title{
A Path Planning Method for Indoor Robots Based on Partial \& Global A-Star Algorithm
}

\author{
WANG Kang-le ${ }^{1}$, DANG Shu-wen ${ }^{1,{ }^{*}}$, HE Fa-jiang ${ }^{1}$, CHENG Peng-zhan $^{2}$ \\ ${ }^{1}$ School of Air Transportation and Flying, Shanghai University of Engineering Science, Shanghai, \\ 201620, China. \\ 2 School of Mechanical Engineering, Shanghai University of Engineering Science, Shanghai, \\ 201620, China. \\ koolewang@163.com, swdang@126.com, mikehfi@163.com, eden0@foxmail.com
}

Keywords: A-Star Algorithm; Indoor Robot; Path Planning; LIDAR

\begin{abstract}
Considering the traditional A*algorithm being difficult to satisfy the limitation of the indoor environment space of the robot. A partial and global A-Star (P\&G-A*) algorithm based on the motion model of indoor robot is proposed. LIDAR sensing system is employed into P\&G-A* algorithm to achieve both local awareness and global optimization. The real environment data is adopted for simulated experiments, and experimental results proved that the path planning time is reduced $13.31 \%$ when comparing with traditional algorithm, and actual driving distance decrease by $15.71 \%$. Furthermore, the robot trajectory is more smoothly after P\&G-A* is applied.
\end{abstract}

\section{Introduction}

The path planning of human is a process of comprehensive analysis of memory information and real-time sensing information in the real world, which is belong to the behavior control in robot technology field. The autonomous path of the robot involves global path planning and local path planning in the indoor environment [1,2]. To solve the problem of indoor robot path planning, several algorithms are commonly used including A-Star search algorithm, probabilistic roadmap method (PRM), dynamic programming method, Dijkstra algorithm, genetic algorithm, particle swarm algorithm, Mathematical planning and so on[3,4].

This paper established the indoor robot path planning model. Meanwhile, to obtain the necessary environmental information planning the LIDAR simulation sensing system is introduced. The P\&G-A*algorithm based on local perception and global optimization is proposed to perform environmental path search. The algorithm is applied to solve the problem of track planning in order to achieve the requirements of the indoor robot to avoid the obstacle and the optimal path to reach the target.

\section{A-Star Algorithm}

The A-Star search algorithm is widely used because it is easy to be calculated and implement. As a heuristic graph search algorithm, A-Star can satisfy the constraints and requirements for track planning. It can theoretically guarantee the convergence of the global optimal solution and the valuation function changes after the search node being extended[5].

In $A^{*}$ method, the search area is transformed into a two-dimensional array, which is a group of square grids and the center of the network is defined as node $(n)$. Each element of the array is a square of the grid. The two dimensional box is defined as a passable node (OPEN table) and a non-passable node (CLOSE table).The general form of the valuation function is as follows[7].

$$
f(n)=g(n)+h(n)
$$

The A*algorithm's flow is shown in Fig.1.Where $f(n)$ is the evaluation function from the initial point 
through the node $n$ to the target point, and $g(n)$ is the actual cost of the initial node to the $\mathrm{n}$ node in the state space. $h(n)$ is the estimate of the optimal path from $n$ to the destination node[8].

The A*algorithm is shown in Fig. 1 as follow. Where $f(n)$ is to evaluate the importance of each node in OPEN table and determine the order of nodes. The OPEN table is used to record nodes that have been calculated but are not expanded, while the CLOSE table is adopted to store the nodes that have been expanded. During all steps of the search process, the nodes in the OPEN table need to be found firstly to calculate the evaluation function, then be added the CLOSE table until the OPEN table is empty .

It seems that the $\mathrm{A} *$ algorithm is theoretically optimal to save the computation time, but the search time will increase because the number of nodes growing more while searching space being enlarged, the application of $\mathrm{A}^{*}$ algorithms is constrained by the size of the indoor environment map.

\section{Application of P\&G-A*Algorithm in Robot Path Planning}

P\&G-A*Algorithm. Although the best path can be obtained in the traditional $A^{*}$ algorithm, the largest number of nodes are involved so that the searching time are scarified. Furthermore, the more A-Star nodes being extended, the lower operation efficiency is deduced.

Considering the problems deduced in traditional $A *$ algorithm, the $P \& G-A * a l g o r i t h m$ is proposed to optimize the search range and reduce the redundant nodes from the robot's local perception system and the robot motion system. The flow chart of the P\&G-A*algorithm is shown in Fig. 2.
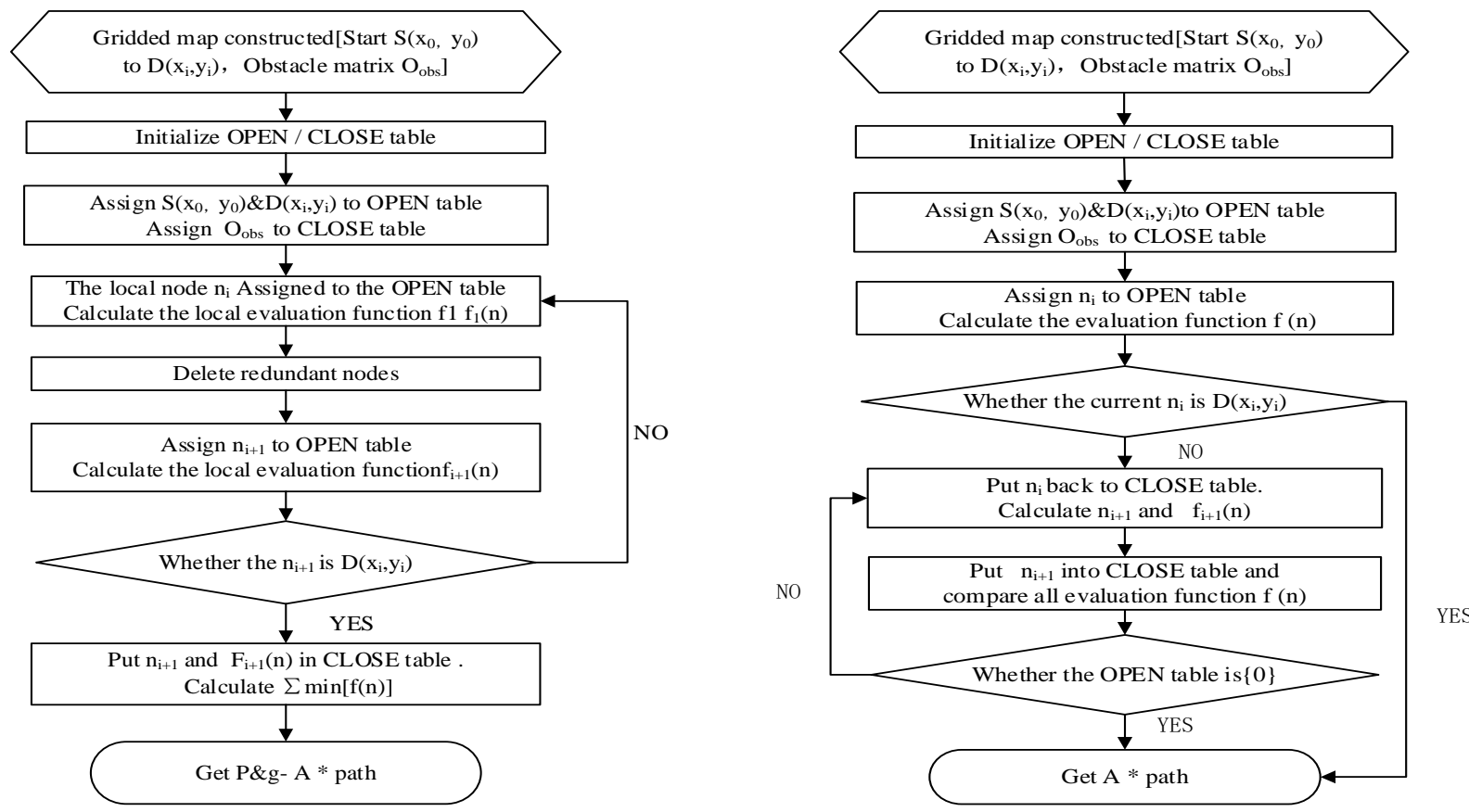

Fig.1. The flow chart of the $\mathrm{A} *$ algorithm

Fig.2. The flow chart of the P\&G-A* algorithm

Track Planning Simulation based on Three Algorithms . In this part, the $100 * 100$ size simulated complex indoor map is established firstly. The starting point is marked as red circle in FIGURE6.The target point is expressed by rectangle, and the obstacle is represented as the bar. The position of the starting and end point of the indoor robot are $(5,5)$ and $(90,90)$ respectively.

The PRM algorithm is composed of two stages such as offline learning stage and online query phase in the path planning problem. As comparison, the PRM method, traditional A* and P\&G-A* algorithm are all applied in simulated experiments as show in Fig.3,4. 


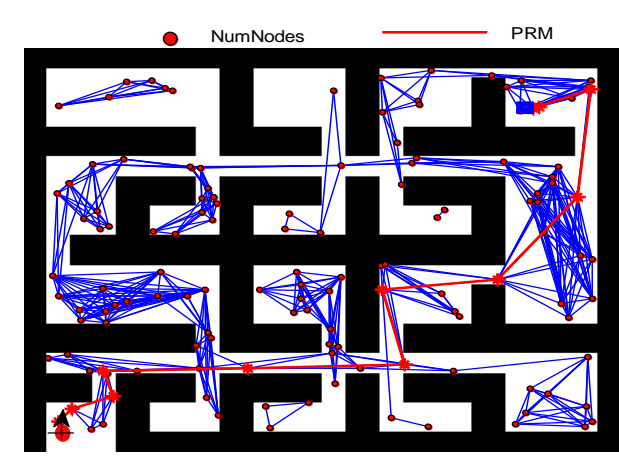

Fig.3 Path planning trajectory based on PRM

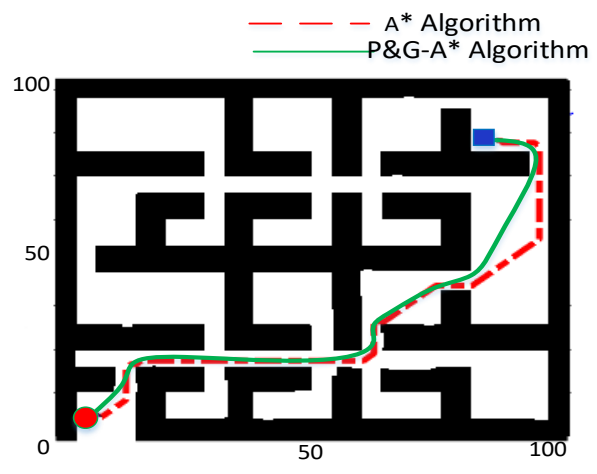

Fig.4 Path planning trajectory comparison between A* withP\&G-A*algorithm

The number of nodes in the OPEN table and the length of the planning path are both compared with the original A-Star algorithm and the P\&G-A*algorithm, and the experimental results is listed in Table 1 as follows.

Table 1 Performance comparison between the A*/PRM and the P\&G-A*algorithm algorithms

$$
\text { Algorithm Number of breakpoints Path distance Time consuming (ms) }
$$

\begin{tabular}{cccc}
\hline PRM & 13 & 315.62 & 14.748 \\
\hline A-Star & 9 & 217.38 & 3.482 \\
\hline Improved A-Star & & 188.45 & 2.935 \\
\hline
\end{tabular}

The number of nodes expanded with the P\&G-A*algorithm is greatly reduced as well as the path cost (the length of the tracking path in this case). The reason for this is that the redundant nodes is deleted in P\&G-A*algorithm. The sensing range of the LIDAR sensor is used as the search radius so that the search step is expended. Thus reducing the expansion of the node and the larger the search step can be effectively optimized for the path and resulting in the path length decreased.

Experiment and Result . The improved algorithm is applied to the indoor robots equipped with LIDAR sensor as shown below. The internal structure of the robot is shown in Fig.5. The Mapping results with $\mathrm{A}^{*}$ (bluedot) and P\&G-A*algorithm (red dot) compared with path planning track is shown in Fig.6 as follow.

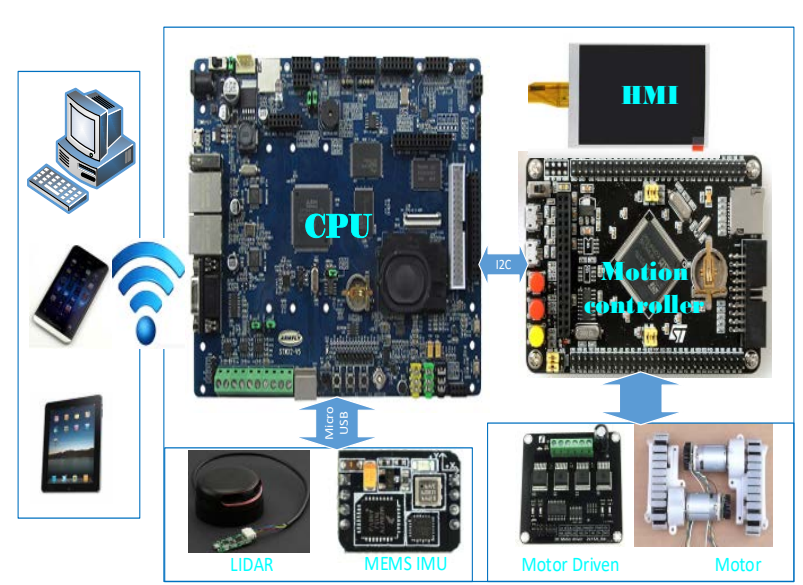

Fig.5 All system and internal structure of the robot

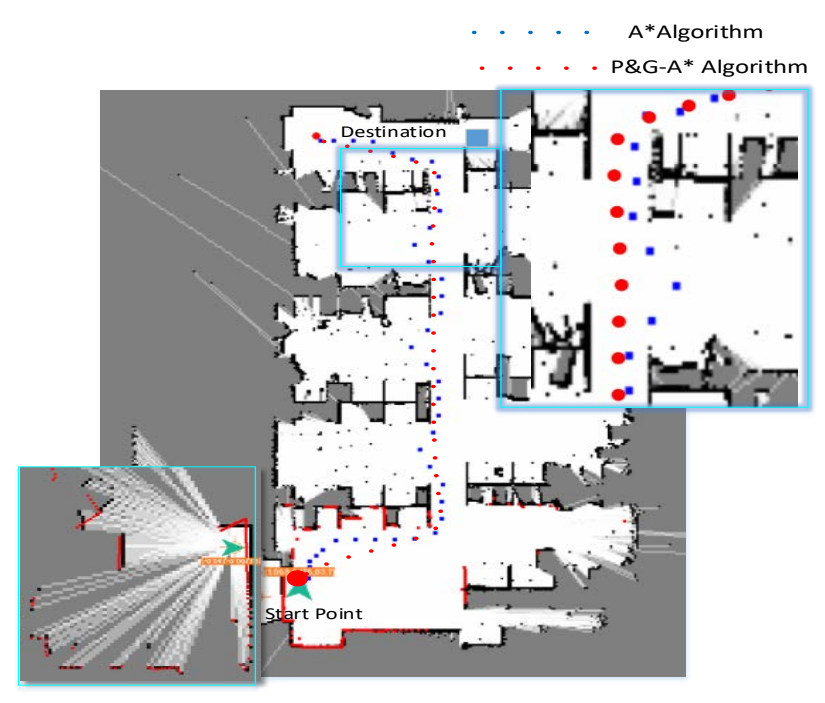

Fig.6 The Mapping results with A*(bluedot)andP\&G-A* algorithm (red dot) compared with path planning track

In the actual environmental test, the blue dotted line is the actual tracking path by using traditional A-Star algorithm. The planned tracking path of the P\&G-A*algorithm is shown in red 
dotted line as shown in the Fig.6. The comparison of track distance and time consuming is listed in Table 2.

Table 2 Experimental environment performance comparison between the $\mathrm{A}^{*}$ and the P\&G-A* algorithm

\begin{tabular}{ccc}
\hline Algorithms & Actual driving distance $(\mathrm{m})$ & Time consuming $(\mathrm{s})$ \\
\hline $\mathrm{A}^{*}$ & 10.09 & 16.89 \\
\hline P\&G-A* & 8.75 & 14.24 \\
\hline Decrease the percentage & $13.31 \%$ & $15.71 \%$ \\
\hline
\end{tabular}

\section{Summary}

A P\&G-A*algorithm based on LIDAR sensor combined the local optimization and global programming is present and applied for indoor robot path planning. The simulation results and the actual test results show that:

(1) The P\&G-A*algorithm combines the local optimization and the global planning to break through the limitation of the minimum turning radius of the traditional A-Star algorithm and is used to overcome the expansion of the number of nodes involved. In the detecting range of laser radar, the incremental patterning path is optimized, which can solve the problem that the computing time increases rapidly while map space increasing.

(2) The actual robot path experiment shows that the P\&G-A*algorithm effectively reduces the path planning time, which is $13.31 \%$ less than the $\mathrm{A}^{*}$, while driving distance is depressed as $15.71 \%$ less than $\mathrm{A}^{*}$.Meanwhile, the path track is more smooth.

\section{References}

[1] Yang Chao. Path Planning of Mobile Robot Based on A* Algorithm [J]. Electronic Technology and Software Engineering, 2015, (24): 119.

[2] Liu Jingkun.Dynamic path planning of indoor mobile robot[D]. Zhengzhou University, 2014.

[3] Mahdi Fakoor, Amirreaz Kosari, Mohsen Jafarzadeh.Humanoid Robot Path Planning With Fuzzy Markov Decision Processes[J]. Journal of Applied Research and Technology.2016.

[4] Li Ji,Sun Xiu Xia.A route Planning's Method for Unmanned Aerial Vehicles Based on Improved a A* Algorithm[J].ACTA Armament ARIL ,2008.

[5] Xia Yan, Sui Yan. Research on optimization of PRM path planning algorithm [J].Applied Science and Technology,2010,37(10):1-5.

[6] Nanaz Fathpour, Lars Blackmore, Yoshiaki Kuwata,ect.Feasibility Studies on Guidance and Global Path Planning for Wind-Assisted Montgolfière in Titan[J]. IEEE Systems Journal ,2014,8(4): 1112 - 1125.

[7] PeiyaoShen,XueboZhang, Yongchun Fang.Essential Properties of Numerical Integration for Time-Optimal Path-Constrained Trajectory Planning[J]. IEEE Robotics and Automation Letters,2017,2(2): 888 - 895.

[8] Jeffrey Delmerico, Elias Mueggler, Julia Nitsch,ect. Active Autonomous Aerial Exploration for Ground Robot Path Planning[J]. EEE Robotics and Automation Letters,2017,2(2): 664 - 671. 